\title{
Validation of a delocalized measurement method of lactate for septic patients
}

\author{
R. Jouffroy ${ }^{1}$, T. Leguillier², M. Boisson², A. Boussaroque², V. Nivet Antoine², JL. \\ Beaudeux ${ }^{2}$, B. Vivien ${ }^{1}$ \\ ${ }^{1}$ Intensive Care Unit, Anesthesiology and SAMU - Necker-Enfants malades hospital, \\ University Paris Descartes - APHP - 149 rue de Sèvres 75015 Paris - France \\ ${ }^{2}$ Biochemistry - Necker-Enfants malades hospital, University Paris Descartes - APHP - \\ 149 rue de Sèvres 75015 Paris - France
}

\section{INTRODUCTION}

Septic shock is defined as a sepsis with hyperlactaemia greater than $2 \mathrm{mM}$ after correction of hypovolemia requiring vasopressors to maintain $\mathrm{MBP}>65 \mathrm{mmHg}$ [1]. It can be observed in pre-hospital emergency medicine (PHEM). The use of a reliable portable device for measuring lactate in PHEM would allow a better evaluation of septic patient facilitating their orientation towards intensive care unit (ICU) or emergency department (ED). This portable delocalized biology device must be validated against the laboratory reference method (NFEN ISO 22870) [2].

The aim of this study was to clarify the validity of a delocalized measure of lactatemia.

\section{METHOD}

We performed a prospective study including 47 patients admitted into ICU for septic shock (CPP number 2015-08-03 SC). Lactate was measured in parallel on 2 samples: one capillary with the portable device (Lactate StatStrip Xpress, Nova Biomedical) and the other venous on a centrifuge tube for plasma analysis (Architect C16000 Abbott Diagnostics).

We evaluated the analytical performance (coefficients of variation (CV) for repeatability and reproducibility evaluated at 2 levels of quality control (QC): 1.6 and $3.6 \mathrm{mM}$ ) and then the concordance between lactate levels measured by the devices and lactate levels measured by laboratory analyzer.

\section{RESULTS}

At the QC concentrations tested, the CVs were in agreement with the limits set by the French Society of Clinical Biology: CV $<3 \%$ for repeatability and $<5 \%$ for reproducibility. An excellent correlation was observed between the 2 measurements: correlation coefficient $\mathrm{R} 2=0.98$, slope $=0.95$ and ordered at the origin $=0.1$. The latter suggested a low positive bias of the device not confirmed by Bland-Altmann graph analysis and graph of the differences

\section{DISCUSSION}

We verified the analytical performance of the device and showed an excellent correlation with the laboratory measurement. The delocalized measure can be used in PHEM in patients with suspected sepsis syndrome. This measure should allow a more accurate and early assessment of their severity in order to improve triage and hospital orientation between ED and ICU.

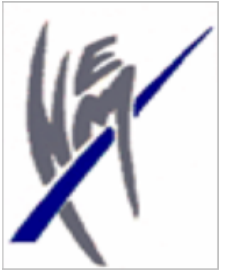

